\title{
Adulteration in Milk: A Growing Concern
}

\author{
Rajib Biswas* \\ Department of Physics, Tezpur University, India
}

Submission: January 02, 2019; Published: January 11, 2019

*Corresponding author: Rajib Biswas, Applied Optics and Photonics Lab, Department of Physics, Tezpur University, Tezpur-784028, Assam, India

Abstract

Adulteration is a rising concern for global community. This mini review offers a brief glimpse of the commonly used adulterants in milk industry. Apart from this, the various methods used to detect variety of adulterants are also highlighted along with recommendations.

\section{Opinion}

Adulteration is alarmingly rising in food industry. Due to lack of awareness as well as effective monitoring mechanism, the intake of adulterated food leads to some undesired consequences such as various diseases to causalities in worst cases. As for example, one of the severely affected industry is the dairy industry; basically, milk products. The global dairy market is estimated to be $\sim 400$ billion U.S dollar. Particularly, the developing nations are facing real challenges although the problem persists in developed nations. It is of consensus that milk is one of the most frequently adulterated foods. Due to explosion of population and urbanization, the demand of milk surpasses the supply. Consequently, the producers and vendors are tempted to adulterate the milk. They take up fraudulent measures to augment the supply. Milk which is adulterated in any form is very much harmful to both adults and children.

Several reports of adulteration in milk have been reported in recent past. The common chemical adulterants, which have been reported in milk, are sodium hydroxide, formalin, hydrogen peroxide, cane sugar, starch, gelatin, synthetic dyes, soap detergents and Urea. Milk contains solids like minerals, lactose and proteins apart from the milk fat content. Urea is a normal constituent of milk and it is a major part (55\%) of the non-protein nitrogen of milk. However for commercial benefits chemicals like urea, caustic soda, refined oil and detergents are used to adulterate the milk. These adulterations decrease the nutritive value of milk and pose a great threat to human health. Every nation has a defined limit of concentration of this component. A cut off limit of urea in milk is normally accepted at $70 \mathrm{mg} / \mathrm{dl}$. The presence of urea above the cut off limit in milk can cause health problems such as indigestion, acidity, ulcers, cancer etc.

In 2008, in China the addition of melamine to infant formula and other milk products led to the death of both infants and others.
Samples of wheat gluten mixed with melamine, presumably to increase the apparent protein contents, were discovered in many U.S. pet food brands in 2007, as well as in the human food supply. Lately, the instances of adulteration with melamine, urea and other substances in milk and milk products have risen and are posing great threat to human health. Besides, processing can also alter the composition of minor constituents, thus adding to the problems in detection. Addition of starch in milk leads to thicken the milk and to increase the 'solid not fat' content of the milk. This in turn deprives the other nutrients present in milk. In the same note, soap powder is added to augment the lather in double toned milk. During transportation of milk, spoilage of milk is an important factor for the suppliers. In order to preserve the milk, urea is added to it. The other adulterants added are hydrogen peroxide and sodium bicarbonate. Hydrogen peroxide and sodium bicarbonate are added to prevent the milk from getting spoiled, sour and to remove the dirt from it.

However, being categorized as neutralizers, their names remain hidden in packaging. A variety of analytical techniques have been developed for the determination of urea, although no single technique is that versatile to provide satisfactory results in all areas. Methods are often categorized as direct or indirect. The term indirect refers to the enzymatic degradation of urea prior to detection. Direct procedures have been defined as those resulting in a colored product, which indicates the presence of urea, but this method cannot give quantitative measurements. Various analytical techniques available to determine urea are based on colorimetric or chromatographic methods, which consume much time to get the species analyzed and are too tedious and expensive to be used. Although lacking the inherent selectivity of the colorimetric technique, UV absorbance measurement also offers a convenient method of detection when coupled with chromatographic or electro-phonetic separation. Infrared spectrometry is another 
technique for the determination of urea, which may be particularly suitable for dairy industry research, where this technique is already employed for the analysis of milk for fats and proteins.

However, multivariate-analysis techniques are required to estimate the contribution of urea from the overlapping absorption bands of other components. The food and fodder products are complex mixtures of chemically diverse compounds, so highly specific and reliable methods are needed for their analysis and milk is one of them. In addition, the govt. agencies should keep a close eye on this so that infringement of defined limit can be easily brought under control. There should be stringent as well as punitive measure for those who violate the norms setup by the regulating agencies. In the same note, large scale awareness is also inevitable in this direction. 